\title{
Potential value of EUS in pancreatic surveillance of VHL patients
}

\author{
Sophie J van Asselt 1,2, Adrienne H Brouwers ${ }^{3, *}$, Hendrik M van Dullemen 4, *, \\ Eric J van der Jagt ${ }^{5}$, Alfons H Bongaerts ${ }^{2,3,+}$, Klaas P Koopmans ${ }^{6}$, Ido P Kema7, \\ Bernard A Zonnenberg ${ }^{8}$, Henri J Timmers' ${ }^{9}$, Wouter W de Herder ${ }^{10}$, Wim J Sluiter ${ }^{1}$, \\ Elisabeth G de Vries ${ }^{2}$ and Thera P Links ${ }^{1}$
}

${ }^{1}$ Department of Endocrinology, ${ }^{2}$ Department of Medical Oncology, ${ }^{3}$ Department of Nuclear Medicine and Molecular Imaging, " Department of Gastroenterology, ${ }^{5}$ Department of Radiology, University of Groningen, University Medical Center Groningen, Groningen, The Netherlands, ${ }^{6}$ Department of Nuclear Medicine and Molecular Imaging, Martini Hospital Groningen, Groningen, The Netherlands, ${ }^{7}$ Department of Laboratory Medicine, University of Groningen, University Medical Center Groningen, Groningen, The Netherlands, ${ }^{8}$ Department of Internal Medicine, University Medical Center Utrecht, Utrecht, The Netherlands, ${ }^{9}$ Department of Medicine, Divisioin of Endocrinology, Radboud University Nijmegen Medical Center, Nijmegen, The Netherlands, and ${ }^{10}$ Department of Endocrinology, Erasmus Medical Center, Rotterdam, The Netherlands *(A H Brouwers and H M van Dullemen contributed equally to this work) ${ }^{\dagger}$ (Deceased)

Correspondence should be addressed to Thera $\mathrm{P}$ Links Email t.p.links@umcg.nl

\begin{abstract}
Background: Patients with von Hippel-Lindau (VHL) disease are prone to develop pancreatic neuroendocrine tumors (pNETs). However, the best imaging technique for early detection of pNETs in VHL is currently unknown. In a headto-head comparison, we evaluated endoscopic ultrasound (EUS) and ${ }^{11} \mathrm{C}$-5-hydroxytryptophan positron emission tomography $\left({ }^{11} \mathrm{C}-5-\mathrm{HTP}\right.$ PET) compared with conventional screening techniques for early detection of pancreatic solid lesions in VHL patients.

Methods: We conducted a cross-sectional, prospective study in 22 patients at a tertiary care university medical center. Patients with VHL mutation or with one VHL manifestation and a mutation carrier as first-degree family member, with recent screening by abdominal computed tomography (CT) or magnetic resonance imaging (MRI) and somatostatin receptor scintigraphy (SRS), were eligible. Patients underwent EUS by linear Pentax echoendoscope and Hitachi EUB-525, and ${ }^{11} \mathrm{C}-5$-HTP PET. Patient-based and lesion-based positivity for pancreatic solid lesions were calculated for all imaging techniques with a composite reference standard.

Results: In 10 of the 22 patients, 20 pancreatic solid lesions were detected: 17 with EUS $(P<0.05$ vs CT/MRI+ SRS), 3 with ${ }^{11} \mathrm{C}-5$-HTP PET, 3 with SRS, 9 with CT/MRI, and 9 with CT/MRI + SRS. EUS evaluations showed solid lesions with a median size of $9.7 \mathrm{~mm}$ (range $2.9-55 \mathrm{~mm}$ ) and most of them were homogeneous, hypoechoic, isoelastic, and hypervascular. Moreover, EUS detected multiple pancreatic cysts in 18 patients with a median of 4 cysts (range 1-30). Conclusions: EUS is superior to CT/MRI + SRS for detecting pancreatic solid lesions in VHL disease. ${ }^{11} \mathrm{C}-5-\mathrm{HTP}$ PET has no value as a screening method in this setting. EUS performs well in early detection of pNETs, but its role in VHL
\end{abstract} surveillance is unclear.

() 2016 European Society of Endocrinology Printed in Great Britain
European Journal of Endocrinology

(2016) 174, 611-620
Published by Bioscientifica Ltd. 


\section{Introduction}

Pancreatic neuroendocrine tumors (pNETs) are uncommon neoplasms with an incidence of 1-10 per 1 million in the general population $(1,2)$. pNETs can occur sporadically or as part of the hereditary multiple tumor syndromes von Hippel-Lindau (VHL) disease and multiple endocrine neoplasia type 1 (MEN1). VHL disease is a dominantly inherited multiple tumor syndrome that results from a germline mutation in the $V H L$ gene located on chromosome 3 . The incidence rate is 1 per 36000 live births. VHL disease can lead to the development of benign and malignant tumors in various organ systems, including hemangioblastomas in the central nervous system, renal cysts and clear cell renal cell carcinomas, pheochromocytomas, pancreatic cysts, and pNETs (reviewed by Lonser et al. (3)). VHL patients have a shorter life expectancy compared with the general population, and $73 \%$ of deaths are related to VHL disease (4). Metastatic clear cell renal cell carcinomas and central nervous system hemangioblastomas are the main cause of VHL-related mortality. Therefore, VHL patients undergo screening to detect early manifestations (3). In patients in whom VHL was diagnosed after 1990, life expectancy was increased by 16 years compared with those diagnosed before 1985, which is likely a result of improved screening strategies and therapeutic options of the last decades (4). Nonfunctional pNETs are present in $10-17 \%$ of the VHL patients $(5,6,7)$, but this prevalence will likely increase due to increased survival rates in VHL.

pNETs in VHL disease can remain indolent for years, but they also have the ability to metastasize. Since the only curative treatment for pNETs is surgery, timely detection and treatment are critical. However, the optimal diagnostic and therapeutic approach is still a matter of discussion. Possible predictors for malignant potential are (i) germline mutation in the VHL gene located on exon 3; (ii) tumor size doubling rate is lesser than 500 days, and (iii) tumor size of $>2 \mathrm{~cm}$ (medium risk) $/>3 \mathrm{~cm}$ (high risk) (6). Another impediment in VHL patients is the presence of both pancreatic benign microcystic serous cystadenomas and pNETs. The differentiation between both entities on radiological imaging can be difficult $(3,6,7,8)$. Currently, the best imaging technique for early detection of pNETs in VHL disease is unknown. For screening of the kidneys, adrenals, and pancreas, yearly abdominal computed tomography (CT) was recommended from 18 years of age (3). The VHL handbook, revised in 2012, recommends yearly abdominal ultrasound and magnetic resonance imaging (MRI) at least every other year from 16 years of age onwards (7, http://www.vhl. org/handbook/vhlhb4). In case of suspicion of a sporadic pNET, the National Comprehensive Cancer Network (NCCN) guideline recommends multiphase CT or MRI, and suggests that somatostatin receptor scintigraphy (SRS) plus circulating tumor markers (serum chromogranin A and plasma pancreatic polypeptide) should be considered [http://www.nccn.org/professionals/physician_gls/pdf/ neuroendocrine.pdf]. In an imaging study carried out in 69 VHL patients with pancreatic solid lesions, abdominal CT was more sensitive for detection of pancreatic lesions than MRI, 6-[F-18]fluoro-L-dihydroxyphenylalanine $\left({ }^{18} \mathrm{~F}\right.$-DOPA), and ${ }^{18} \mathrm{~F}$-fluorodeoxyglucose $\left({ }^{18} \mathrm{~F}-\mathrm{FDG}\right)$ positron emission tomography (PET) (8). The disadvantage of CT, however, is its radiation burden, which may be even more undesirable in genetically vulnerable VHL patients.

Endoscopic ultrasound (EUS) and ${ }^{11} \mathrm{C}-5$-hydroxytryptophan $\left({ }^{11} \mathrm{C}-5\right.$-HTP) PET are of potential interest in the search for sensitive and specific pNET imaging in VHL. In a retrospective control study, Rösch et al. (9) showed that EUS identified $82 \%$ of histologically proven sporadic functional pNETs that were undetectable by trans-abdominal ultrasound and CT. No such studies are available in VHL patients. However, in the hereditary multiple tumor syndrome MEN1, EUS performs excellent in pNET detection with a high sensitivity $(10,11,12,13,14,15)$. In addition, ${ }^{11} \mathrm{C}-5$-HTP PET could potentially provide insights into the neuroendocrine nature of a pancreatic lesion. ${ }^{11} \mathrm{C}-5-\mathrm{HTP}$ is a precursor of serotonin, which neuroendocrine cells can take up, decarboxylate into ${ }^{11} \mathrm{C}$-serotonin, and store in vesicles (16). In 23 patients with advanced pNETs with a total of 294 lesions, ${ }^{11} \mathrm{C}-5$-HTP PET was more sensitive in detecting lesions than SRS and ${ }^{18}$ F-DOPA PET (17). ${ }^{11} \mathrm{C}$-HTP PET and CT performed equally well at a lesionbased level (sensitivity of $67 \%$ and $68 \%$ respectively), and proved better compared with ${ }^{18} \mathrm{~F}$-DOPA PET (sensitivity $41 \%$ ) and SRS (sensitivity 46\%) (17). The aim of this study was therefore to evaluate EUS and ${ }^{11} \mathrm{C}-5$-HTP PET in a head-to-head comparison relative to standard screening for the detection of pNETs in VHL patients.

\section{Subjects and methods}

\section{Patients}

In this cross-sectional, prospective study, patients under standard surveillance in the VHL centers of the Erasmus MC Rotterdam, University Medical Centers Utrecht 
and Groningen were referred to the University Medical Center Groningen for participation in the study. The patients were included between February 2009 and August 2011. The eligible patients were those with a VHL mutation or with one VHL manifestation and a mutation carrier as first-degree family member of age $\geq 18$ years. Those excluded were pregnant patients or patients with known alcohol abuse and/or chronic pancreatitis. The study was approved by the Medical Ethics Committee of Groningen on August 25, 2008, and all patients gave written informed consent. The study was registered at the Dutch trial registry under http://www.trialregister.nl/ trialreg/index.asp (NTR1668).

Before study entry, a standard workup for screening had to be performed in the patient's own center, this included SRS within 6 months, and abdominal CT or MRI and serum/plasma tumor markers within 4 months. With radioimmunoassays, serum levels of chromogranin A (CgA-React, Cis Bio International, Gif-sur-Yvette, France, upper limit of normal $100 \mu \mathrm{g} / \mathrm{L}$ ) and plasma levels of pancreatic polypeptide (Eurodiagnostica, Nijmegen, the Netherlands, upper limit of normal $100 \mathrm{pmol} / \mathrm{L}$ ) were determined. Since the use of proton pump inhibitors can lead to spurious elevation of chromogranin A (18), patients on proton pump inhibitors were excluded from the analysis of these levels. Values greater than upper limit of normal were considered as overproduction.

\section{CT/MRI}

The patients underwent abdominal CT or MRI, as recommended by the treating physician. CT and MRI scans were performed as described previously (15). The lesions were recorded as pancreatic solid lesions when they were observed as hyper-attenuating lesions on the arterial or portal venous phase-contrast images. On MRI, solid lesions were considered to have low intensity on $\mathrm{T} 1$ sequences and high intensity on $\mathrm{T} 2$ sequences compared with pancreatic tissues (19). CT and MRI reports were reviewed by a radiologist (EJvdJ) blinded for the clinical information. In case of discrepancy between the radiologist of the referral and research centers, a second radiologist (AHHB) reviewed these particular scans, and consensus was reached after discussion between EJvdJ and AHHB.

\section{SRS}

Twenty-four hours after i.v. administration of 200 $\mathrm{MBq}^{111}$ In-pentetreotide (Octreoscan; Mallinckrodt, Petten, the Netherlands), planar whole-body and abdominal 3D single-photon-emission computed tomography (SPECT (all patients)/CT $(n=8)$ ) images were obtained (20). The SRS scans were reviewed by a nuclear medicine physician (AHB). In case of discrepancy between the nuclear medicine physician of the referral and research centers, a second nuclear medicine physician (KPK) reviewed the SRS, and after discussion between AHB and KPK, consensus was reached.

\section{EUS and ${ }^{11} \mathrm{C}-5-\mathrm{HTP}$ PET}

If possible, EUS and ${ }^{11} \mathrm{C}-5$-HTP PET were executed on the same day. EUS was performed as described previously (15). The presence of vascularity was assessed with power Doppler and elasticity or rigidity by elastography imaging (21). The patients underwent conscious sedation, and EUS was performed by one endoscopist (HMvD) blinded for other imaging results. The number and location of pancreatic findings were recorded on a standardized record form. The pancreatic lesions with anechogenic appearance plus an enhanced ultrasound signal were interpreted as simple cysts. The lesions with a honeycomb pattern were classified as microcystic serous cystadenomas (22). Other focal lesions in the pancreas were considered as solid lesions. Fine-needle aspiration (FNA) with a 22 or 25 G needle with a stylet (Sono Tip II 22 or 25 Gauge Medi-Globe $\mathrm{GmbH}$, Germany) was performed on solid lesions if cytological confirmation of pNET was desired by the referred physician. A cytotechnologist was present during the FNA procedure for on-site assessment of the obtained material.

${ }^{11} \mathrm{C}$-5-HTP PET scans were performed as described previously (17), either using an ECAT HR + PET camera with a spatial resolution of $\sim 10 \mathrm{~mm}(n=11)$ or using a Siemens Biograph mCT camera (PET/CT 64 slices) (Siemens) with a spatial resolution of $\sim 8 \mathrm{~mm}(n=11)$ after reconstruction and post-processing. Maximum standardized uptake value $\left(S U V_{\max }\right)$ was assessed for each lesion. PET scans were independently and randomly interpreted by two nuclear medicine physicians (AHB, KPK) blinded for all clinical information and other imaging. The number and location of positive lesions were recorded on a standardized record form. In discrepant cases, consensus was reached between AHB and KPK.

The results of the four imaging modalities were discussed in a multidisciplinary team, which consisted of a radiologist (EJvdJ), nuclear medicine physician (AHB), 
gastroenterologist (HMvD), endocrinologist (TPL), and the clinical trial doctor (SJvA). The pancreatic solid lesions found on both EUS and ${ }^{11} \mathrm{C}-5$-HTP PET were matched with the CT/MRI + SRS.

\section{Statistical analysis}

A total of 20 patients were estimated to be required to demonstrate additional or new lesions in $25 \%$ of the patients with ${ }^{11} \mathrm{C}-5$-HTP PET and/or EUS. McNemar's test was used for comparison with $80 \%$ power and 5\% twosided significance levels.

Analysis was performed at the levels of individual patients and individual lesions. SRS and ${ }^{11} \mathrm{C}-5$-HTP PET scan are whole-body imaging modalities, whereas CT/ MRI cover the abdominal area and EUS the pancreatic region only. Therefore, EUS was compared with other imaging modalities for detecting tumors in the pancreatic region only. The percentage of visualized solid lesions was calculated for all four imaging techniques by using a composite reference standard. This standard included the sum of the four imaging outcomes. McNemar's test was used to compare the yield of the four imaging techniques. A $P$ value $<0.05$ was considered to be statistically significant.

Table 1 Patient characteristics $(n=22)$ of VHL patients included in this study.

\begin{tabular}{|c|c|}
\hline Characteristics & Value \\
\hline Female/male $(n)$ & $12 / 10$ \\
\hline Age in years median/(range) & $41 /(21-64)$ \\
\hline \multicolumn{2}{|l|}{ VHL germline mutation $(n)$ : } \\
\hline c.-89-?_c.297+?del & 11 \\
\hline c. $500 \mathrm{G}>\mathrm{A}$ & 3 \\
\hline c.-213-?_463+?del & 2 \\
\hline c. $463+2 \mathrm{~T}>$ C p. (?) & 1 \\
\hline c.259_260insA & 1 \\
\hline c. $497 \mathrm{~T}>\mathrm{C}$ & 1 \\
\hline c.509T >A & 1 \\
\hline c. $241 \mathrm{C}>\mathrm{T}$ & 1 \\
\hline *46,XX.ish del(3)(p25-26p25-26)(cos11-) & 1 \\
\hline \multicolumn{2}{|l|}{ Earlier pancreatic surgery $(n)$} \\
\hline Yes/no & $0 / 22$ \\
\hline \multicolumn{2}{|l|}{ Abdominal conventional imaging $(n)$} \\
\hline MRI & 11 \\
\hline $\mathrm{CT}$ & 11 \\
\hline \multicolumn{2}{|l|}{ Serum chromogranin A (mg/L; $n=19)$} \\
\hline Median/range & $38 /(23-118)$ \\
\hline \multicolumn{2}{|l|}{ Plasma pancreatic polypeptide (pmol/L; $n=22)$} \\
\hline Median/range & $58 /(22-234)$ \\
\hline
\end{tabular}

*According to archaic mutation analysis.

$\mathrm{VHL}$, von Hippel-Lindau; MRI, magnetic resonance imaging; $\mathrm{CT}$, computed tomography.

\section{Results}

\section{Patient characteristics}

Of the 31 eligible patients, 9 patients declined participation. Patient characteristics of the remaining 22 VHL patients included in this study are presented in Table 1 . All patients had a proven VHL germline mutation.

\section{Conventional screening}

CT/MRI findings were positive in 7 patients, which detected 9 pancreatic solid lesions. Focal pancreatic uptake on SRS was present in 3 patients, visualizing 3 lesions with increased uptake corresponding with 3 pancreatic solid lesions detected with CT/MRI.

Of the 22 patients, 6 patients had elevated plasma pancreatic polypeptide with a median of $131 \mathrm{pmol} / \mathrm{L}$ (range 115-234 pmol/L). Evaluation of serum chromogranin A in 19 patients showed that one patient had a marginally elevated level of $118 \mu \mathrm{g} / \mathrm{L}$. In total, 7 patients had elevated

Table 2 EUS characteristics of pancreatic solid lesions $(n=17)$ and cysts $(n=139)$.

\begin{tabular}{|c|c|c|}
\hline Characteristics & Solid lesions $(n)$ & Cystic lesions $(n)$ \\
\hline \multicolumn{3}{|l|}{ Localization: } \\
\hline Pancreatic head & 11 & 56 \\
\hline Pancreatic body-tail & 6 & 83 \\
\hline \multicolumn{3}{|l|}{ Lesion size } \\
\hline$>1 \mathrm{~cm}$ & 7 & 47 \\
\hline$\leq 1 \mathrm{~cm}$ & 10 & 92 \\
\hline \multicolumn{3}{|c|}{ Connection pancreatic duct } \\
\hline Yes & 6 & 5 \\
\hline No & 11 & 74 \\
\hline Unknown & 0 & 60 \\
\hline \multicolumn{3}{|l|}{ Echogenic pattern } \\
\hline Hyperechoic & 0 & 0 \\
\hline Hypoechoic & 17 & 0 \\
\hline Anechoic & 0 & 139 \\
\hline \multicolumn{3}{|l|}{ Ultrasonographic texture } \\
\hline Homogeneous & 11 & 133 \\
\hline Heterogeneous & 6 & 6 \\
\hline \multicolumn{3}{|l|}{ Power Doppler signal } \\
\hline Positive & 10 & * \\
\hline Negative & 7 & * \\
\hline \multicolumn{3}{|l|}{ Elastography } \\
\hline Rigid & 2 & * \\
\hline Isoelastic & 11 & * \\
\hline Unknown & 4 & * \\
\hline \multicolumn{3}{|l|}{ Honeycomb structure } \\
\hline Yes & * & 4 \\
\hline No & * & 135 \\
\hline
\end{tabular}

*Indicates that characteristic is not applicable.

Note that the patient in whom almost the total pancreas was replaced by cysts has not been evaluated for cystic lesion characteristics. 
tumor markers with 6 patients exhibiting not more than a 2 -fold elevation. Of these 7 patients, 3 patients (43\%) had pancreatic solid lesions on conventional imaging.

\section{EUS and ${ }^{11} \mathrm{C}-5$-HTP PET}

EUS detected 17 pancreatic solid lesions with a median size of $9.7 \mathrm{~mm}$ (range $2.9-55 \mathrm{~mm}$ ) in 10 patients. Most of the lesions were homogeneous and hypoechoic on EUS with an isoelastic consistency and hypervascular. In addition, in 18 patients 169 pancreatic cysts were found. The EUS characteristics of both cystic and solid lesions are presented in Table 2.

Only 4 patients had no simple pancreatic cysts or microcystic serous cystadenomas, 12 had 1-10 simple cysts, 4 had more than 10 simple cysts, 3 had microcystic serous cystadenoma(s), and 1 patient had almost the complete pancreas replaced by at least 30 cysts. In patients with cysts, the median number of cysts was 4 (range 1-30) per patient (Fig. 1). In patients with pancreatic solid lesions, the median number of cysts was 1 (range $0-9$ ).

${ }^{11} \mathrm{C}-5$-HTP PET detected pancreatic lesions in only 1 patient, which were scanned on the ECAT HR+ camera. In this patient, 3 pancreatic lesions were visualized with ${ }^{11} \mathrm{C}-5$-HTP PET, with a $S U V_{\max }$ ranging from 4.3 to 4.9 . One lesion was confirmed by CT and 2 lesions by EUS (Fig. 2).
Moreover, ${ }^{11} \mathrm{C}-5$-HTP PET detected 15 extra pancreatic focal lesions in 7 patients (Supplementary table 1, see section on supplementary data given at the end of this article) (Fig. 3).

During the EUS procedure, FNA was performed on 6 patients who had 7 solid lesions with a median of 2 passes (range 2-6). The median size was $17 \mathrm{~mm}$ (range 7.2-55), of which 3 lesions were $<10 \mathrm{~mm}$. In the cell materials from 2 lesions of 2 patients, tumor cells were detected. Morphological analysis showed moderate to cell-rich material present in large sheets and rounded clusters of tumor cells with anisonucleosis, visible nucleoli, and 'frothy' cytoplasm. Immunocytochemical staining was available for one sample and was positive for CK8/18, CK19, CD56 chromogranin, and synaptophysin. Ki67 was positive in a few cells. One of these patients underwent surgery and the other patient remained for follow-up in the VHL surveillance.

In total, 3 patients underwent pancreatic surgery because of large solid lesions suspicious of pNET: the first patient had 1 solid lesion of $5 \mathrm{~cm}$ visible on MRI, SRS, and EUS; the second patient had 1 lesion of $2 \mathrm{~cm}$ visible on MRI, SRS, and EUS + confirmation by cytology; and the third patient had 2 lesions of 4 and $3 \mathrm{~cm}$, both visible on CT and 1 lesion visible on EUS. All 3 patients underwent pylorus preserving pancreaticoduodenectomy. All lesions were hypervascular and inhomogeneous based

A
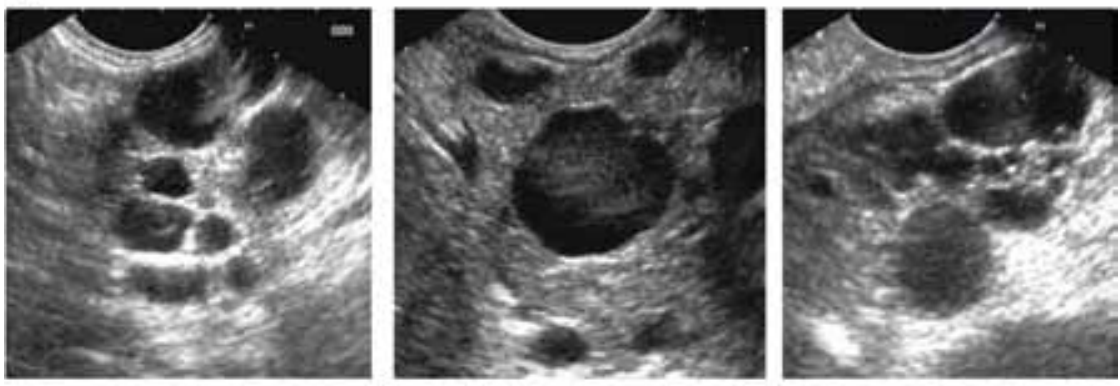

B
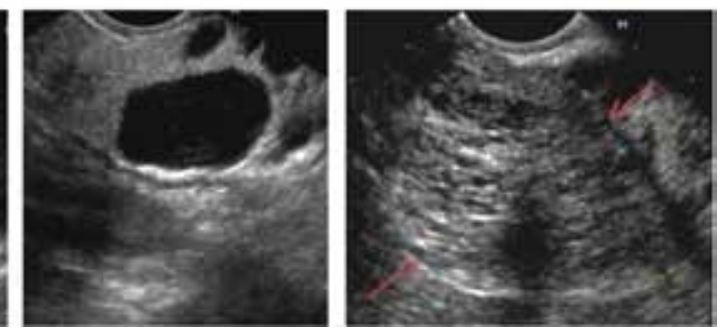

\section{Figure 1}

EUS images of pancreatic cysts of two VHL patients. (A) A 28-year-old man with multiple pancreatic cysts in the entire pancreas, ranging in size from 0.5 to $1.2 \mathrm{~cm}$. The strongly enhanced ultrasound signal (white regions) is the characteristic of the cysts. (B) A 36-year-old woman with multiple cysts in the entire pancreas and (red arrows) a $4.5 \mathrm{~cm}$ microcystic serous cystadenoma with characteristic honeycomb structure in the pancreatic body-tail region. 
A

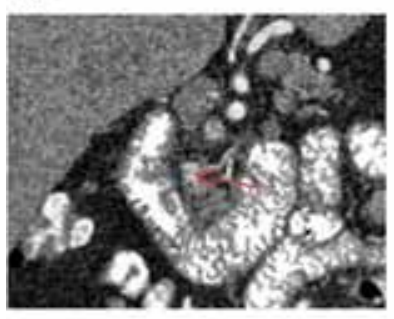

B
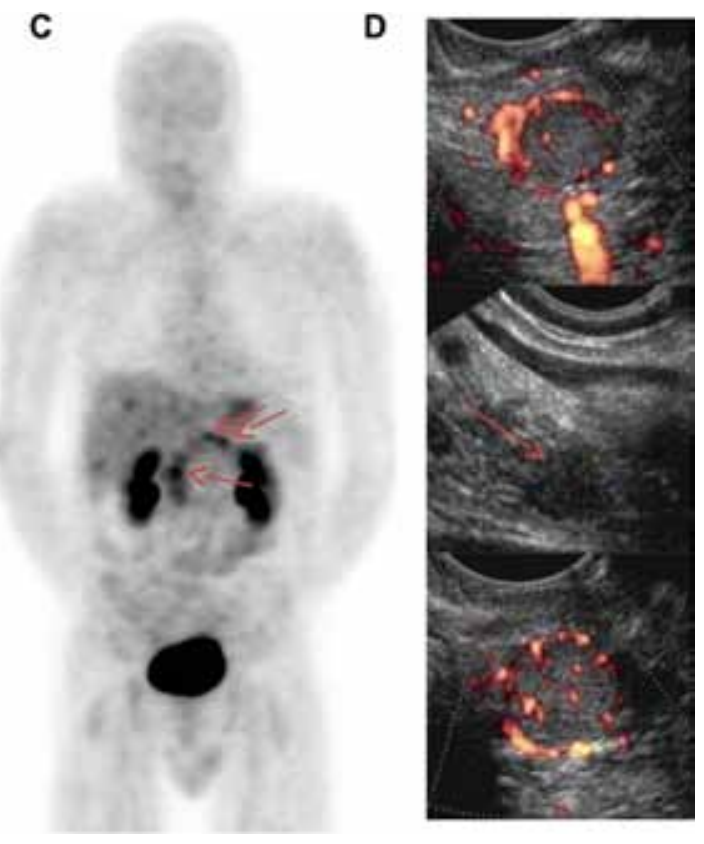

\section{Figure 2}

(A) Coronal images of CT scan, (B) SRS SPECT image, (C) maximum intensity projection image of 11C-5-HTP PET, and (D) ultrasound images of pancreatic lesions visualized with EUS. In total 4 pancreatic solid lesions were detected. With CT, 1 lesion was found. The SRS was negative. 11C-5-HTP PET

on EUS. In the first 2 patients, histology confirmed the diagnosis of NET. However in the third patient, histology showed that both lesions were microcystic serous cystadenomas.

\section{EUS and ${ }^{11} \mathrm{C}-5$-HTP PET compared with conventional screening}

At a patient-based level, pancreatic solid lesions were found in 10 patients: EUS was positive in $100 \%,{ }^{11} \mathrm{C}-5$-HTP PET in $10 \%$, SRS in 30\%, and CT/MRI in 70\%. At a lesion-based level, 20 pancreatic solid lesions were found with a median size of $9.7 \mathrm{~mm}$ (range 2.9-55 $\mathrm{mm}$ ). EUS detected 17 pancreatic solid lesions, ${ }^{11} \mathrm{C}-5$-HTP PET 3, CT/MRI 9, SRS 3, and CT/MRI + SRS detected 9. EUS detected the most solid lesions compared with CT/MRI, SRS, and CT/MRI + SRS $(P<0.05)$ (Table 3$)$. In total, 12 solid lesions were found in the pancreatic head and 8 in the body-tail region, of which EUS detected 11 in the pancreatic head (92\%) and 6 in the body-tail region (75\%). EUS did not detect 3 solid lesions: 2 of these solid lesions were only visualized with CT of which 1 turned out to be a $\sim 3 \mathrm{~cm}$ serous cystadenoma at surgery. Another lesion was only visualized with ${ }^{11} \mathrm{C}-5$-HTP PET. detected 3 lesions: 1 in the pancreatic head and 2 in the body-tail region. EUS detected 2 hypoechoic solid lesions in the pancreatic head and 1 in the body-tail region ranging from 9.7-14.1 mm. Two solid lesions showed hypervascularity with power Doppler.

Of the 9 solid lesions $>1 \mathrm{~cm}, 8$ were detected with EUS, 1 with ${ }^{11}$ C-5-HTP PET, 7 with CT/MRI, 3 with SRS, and 7 with CT/MRI + SRS (Table 3). Seven patients had pancreatic solid lesions visualized on the conventional imaging techniques CT/MRI + SRS, in which EUS found 6 additional lesions. ${ }^{11} \mathrm{C}-5$-HTP PET detected 2 additional lesions in these patients, but missed 8 , which were identified with conventional imaging. However, following resection, 2 of these 8 solid lesions that were ${ }^{11} \mathrm{C}-5-\mathrm{HTP}$ PET negative turned out to be serous cystadenomas.

Of the 15 patients without pancreatic solid lesions on $\mathrm{CT} / \mathrm{MRI}+\mathrm{SRS}$, none had focal ${ }^{11} \mathrm{C}-5$-HTP uptake in the pancreas. In 3 of these patients, 4 solid lesions were found with EUS: all were $<1 \mathrm{~cm}$. Detailed information of each solid lesion suspicious of pNET is shown in Supplementary table 2 (see section on supplementary data given at the end of this article).

Seven patients had elevated tumor markers, of whom 3 patients had only pancreatic cysts, 1 patient had multiple cysts, and 1 pancreatic solid lesion $<1 \mathrm{~cm}$ detected with EUS, 1 patient had 4 pancreatic solid lesions detected with ${ }^{11} \mathrm{C}-5-\mathrm{HTP}$ PET, EUS and/or CT, and 2 patients had pancreatic cysts and NETs detected by histology. 

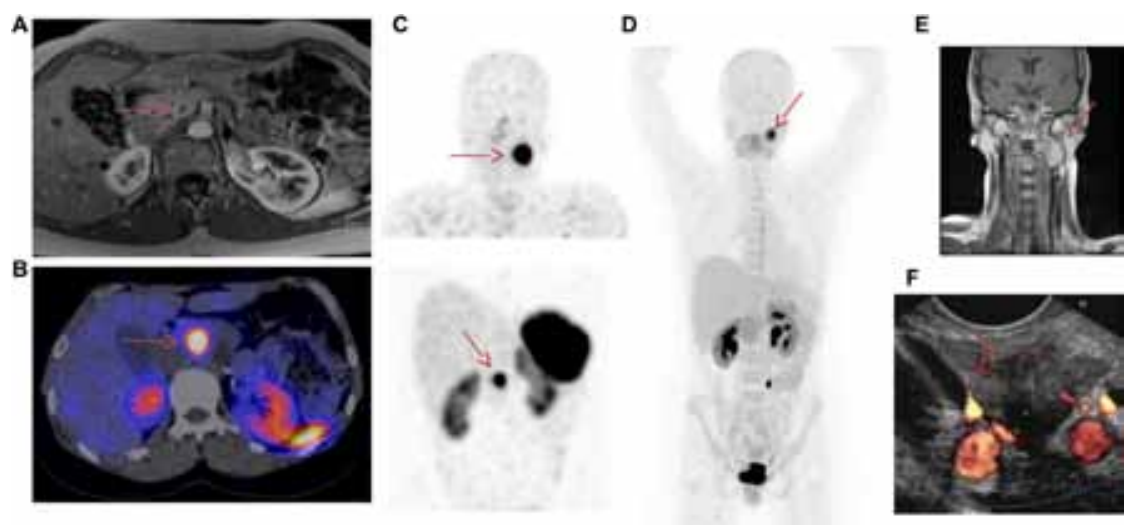

\section{Figure 3}

(A) Axial image of abdominal MRI (T1 weighted with contrast), (B) axial image of SRS fused with low-dose CT,

(C) SRS coronal SPECT image of head and abdominal region,

(D) coronal maximum intensity projection of 11C-5-HTP PET,

(E) coronal MRI of head and neck regions, and

(F) EUS image of the pancreas. On abdominal MRI, 1 pancreatic lesion was found, corresponding with

\section{Discussion}

In this head-to-head comparison of EUS, ${ }^{11} \mathrm{C}-5$-HTP PET, and standard screening techniques CT/MRI and SRS for pNET, EUS was found to be superior for detecting solid pancreatic lesions in VHL patients. ${ }^{11} \mathrm{C}-5$-HTP PET had no value in this screening setting. We have shown for the first time in this rare inherited disease that EUS is a useful method for detecting pancreatic solid lesion. EUS can be executed without radiation exposure, and it allows obtaining cytology by FNA for NET confirmation. Some possible disadvantages of EUS are its invasive character that needs conscious sedation and its operator dependence. However, EUS combined with conscious sedation is proven to be well tolerated (22). Therefore, in our opinion, care for VHL patients should be organized in VHL expertise centers, including an endoscopist experienced in pNETs and VHL disease.
1 focal lesion on SRS. Moreover on SRS, a focal lesion was visualized in the neck region. 11C-5-HTP PET only showed a lesion in the neck at the same location. MRI of the neck confirmed a paraganglioma. With EUS, the $17 \mathrm{~mm}$ pancreatic solid lesion was identified. Cell material obtained by EUS-FNA confirmed the diagnosis of a NET.

Furthermore, with EUS we could identify various lesion characteristics. Most often pancreatic solid lesions were hypoechoic, homogeneous, had an isoelastic consistency and were hypervascular based on power Doppler. Based on EUS combined with power Doppler, these characteristics might help to identify the characteristics of pNETs. Besides solid lesions, we also detected serous cystadenomas with EUS. Serous cystadenomas of the pancreas include microcystic serous cystadenoma, serous oligocystic, and ill-demarcated adenomas as well as macrocystic serous cystadenoma (23). In our series, most cysts were macrocystic serous cystadenomas either "simple cysts", recognized by an anechoic appearance. On EUS, 3\% of the cysts had a classic honeycomb structure, which is the characteristic for microcystic serous cystadenomas.

In VHL, the differentiation between pNETs and serous cystadenomas can be difficult, because serous

Table 3 All patients with pancreatic solid lesions on imaging $(n=10)$.

\begin{tabular}{l}
\hline Imaging modality \\
\hline $\mathrm{CT} / \mathrm{MRI}$ \\
$\mathrm{SRS}$ \\
$\mathrm{CT} / \mathrm{MRI}+\mathrm{SRS}$ \\
${ }^{11} \mathrm{C}-5-\mathrm{HTP} \mathrm{PET}$ \\
$\mathrm{EUS}$ \\
\hline
\end{tabular}

\begin{tabular}{c}
\hline $\boldsymbol{n}(\%)$ of patients \\
\hline $7(70)$ \\
$3(30)$ \\
$7(70)$ \\
$1(10)$ \\
$10(100)$ \\
\hline
\end{tabular}

\begin{tabular}{c}
\hline $\boldsymbol{P}$ value \\
\hline- \\
- \\
- \\
$*<0.05$ \\
$* 0.25$ \\
\hline
\end{tabular}

\begin{tabular}{c}
\hline $\boldsymbol{n}(\%)$ of lesions \\
\hline $9(45)$ \\
$3(15)$ \\
$9(45)$ \\
$3(15)$ \\
$17(85)$ \\
\hline
\end{tabular}

\begin{tabular}{c}
\hline$P$ value \\
\hline- \\
- \\
- \\
$* 0.11$ \\
${ }^{*}<0.05$ \\
\hline
\end{tabular}

\begin{tabular}{c}
\hline $\boldsymbol{n}(\%)$ of lesions $>\mathbf{1} \mathbf{~ c m}$ \\
\hline $7(78)$ \\
$3(38)$ \\
$7(78)$ \\
$1(11)$ \\
$8(89)$ \\
\hline
\end{tabular}

\begin{tabular}{l}
\hline$P$ value \\
\hline- \\
- \\
- \\
$* 0.13$ \\
$* 1$
\end{tabular}

*11 C-5-HTP PET and EUS compared with CT/MRI + SRS.

$\mathrm{CT}$, computed tomography; MRI, magnetic resonance imaging; SRS, somatostatin receptor scintigraphy; ${ }^{11} \mathrm{C}-5-\mathrm{HTP}$ PET, ${ }^{11} \mathrm{C}-5$-hydroxytryptophan positron emission tomography; EUS, endoscopic ultrasound. 
cystadenomas appear also as solid lesions. In our study, 2 lesions with a solid appearance on EUS and CT/MRI suspicious of pNET were shown to be serous cystadenomas by means of histology. Over diagnosis of pNET in VHL has also been reported by others $(6,7,8)$. In our study, one lesion turned out to be a serous cystadenoma, which appeared clearly hypervascular based on EUS combined with power Doppler. This indicates that a hypervascular nature of pancreatic solid lesions in VHL disease does not exclude the existence of a serous cystadenomas. Others also reported cases of pancreatic serous cystadenomas having hypervascular appearances on anatomical imaging $(24,25,26)$. In this situation, molecular imaging may be able to differentiate between pNET and serous cystadenoma. However, if molecular imaging is negative, it might be due to the fact that the solid component of the cystic pNET is too small for detection.

To avoid unnecessary surgery, making a distinction between pNET and serous cystadenoma is warranted in VHL disease. An advantage of EUS is it potentially allows cytology to be obtained by FNA. However, we obtained adequate material in only 2 out of 7 lesions. This may partly be due to the fact that 3 of the 7 lesions were $<10 \mathrm{~mm}$. In a retrospective study in 15 patients using EUS FNA for confirming the diagnosis of pNET, the yield was $53 \%$ (27). Similar to our series, samples were often hemorrhagic with a low cell yield (27). Other retrospective series reported higher accuracy rates of $90-93 \%$, but it is unclear how patients were selected in these studies $(28,29)$. For FNA we used both 22 and 25 Gauge needles. In a recent meta-analysis data of EUS-guided FNA, pancreatic masses were evaluated using 19, 22, and 25 Gauge needles. With respect to accuracy, number of passes, or complication, 22 and 25 Gauge performs equal. Data regarding 19 Gauge needles are limited and did not show the evidence of improved outcomes relative to 22 and 25 Gauge needles (30). However, this meta-analysis is not specific for pNET.

Instead of obtaining cytological material, it is also possible to obtain tissue samples by EUS-guided fineneedle tissue acquisition. In a recent prospective study, 30 patients with pancreatic lesions suspected of sporadic pNET underwent fine-needle tissue acquisition with a 19 Gauge needle, without complications (31). In 93\% of patients with lesion size ranging from 7 to $100 \mathrm{~mm}$, the NET diagnosis could be confirmed (31). In VHL disease, obtaining tissue samples instead of cell material might also improve the yield.

In general, pNETs have the ability to produce hormones or amines, which provide various options for specific imaging by using this metabolic pathway. In 18 patients with advanced NETs, ${ }^{11} \mathrm{C}$-5-HTP PET was superior for lesion detection compared with CT (32). In addition, we showed that ${ }^{11} \mathrm{C}-5$-HTP PET is a more sensitive technique compared with ${ }^{18} \mathrm{~F}$-DOPA PET and SRS in patients with advanced pNET (17). Adding CT resulted in a slight improvement of sensitivity (17). However, in the current VHL study, ${ }^{11} \mathrm{C}-5$-HTP PET showed no added value for early detection of primary pNETs in VHL patients. A possible limitation is the use of two different scanner systems in our study: ECAT $\mathrm{HR}+$ camera and PET/CT with a resolution of $\sim 10 \mathrm{~mm}$ and $\sim 8 \mathrm{~mm}$, respectively. However, only in one patient ${ }^{11} \mathrm{C}-\mathrm{HTP}$ PET was positive and showed (three) pancreatic lesions. This patient was scanned on the, older, ECAT HR+ camera. Therefore, we do not think that this has greatly influenced the results and major conclusions of our study.

Besides pancreatic lesions, ${ }^{11} \mathrm{C}-5$-HTP-PET found 15 other VHL manifestations, including hemangioblastomas, renal cell carcinomas, and paraganglioma. Like pNETs, paragangliomas are able to take up and decarboxylate amine precursors (33). This property has not been identified in renal cell carcinomas and hemangioblastomas. Nevertheless, in both tumor types immunohistochemical studies showed positive staining of specific neuroendocrine markers, including neuron-specific enolase and synaptophysin $(34,35)$, indicating the presence of neuroendocrine properties of these cells. This might clarify visualization with ${ }^{11} \mathrm{C}-5$-HTP PET.

In our study, the value of SRS was limited, because only $15 \%$ of pancreatic solid lesions could be visualized. Another study showed a SRS patient-based positivity of $59 \%$ in 27 VHL patients with pNETs with a median size of $30 \mathrm{~mm}$ (range 10-80 mm) (36). In our study, an explanation for the lower yield of SRS is possibly due to median size of the solid pancreatic lesions, which was $\sim 3$-fold smaller compared with the study of Corcos et al. Altogether, this suggests that SRS is not useful for screening pNET in VHL disease. Nowadays, the somatostatin receptors can be imaged with PET tracers, resulting in an increase in resolution and lesion detectability $(37,38,39)$. Also, these PET tracers have a higher affinity for binding to the somatostatin receptors, potentially also resulting in a better sensitivity (37). There are several studies suggesting the use of PET in pNET screening $(40,41)$. However, in this specific VHL patient population and setting, it is undefined what the merits of PET with somatostatin analogues are.

In our series, 2 out of 4 patients with a cytologically/ histologically confirmed NET had elevated serum chromogranin A or plasma pancreatic polypeptide levels. In total, 7 patients had elevated tumor markers, which were 
marginally increased in 6 patients (not more than 2 times the upper limit of normal). The biological and analytical variations of these markers are not known, making it difficult to interpret these findings. Moreover, false-positive values can occur in a variety of clinical conditions. Elevated chromogranin A levels can occur because of using proton pump inhibitors; renal or hepatic insufficiency; and elevated pancreatic polypeptide levels due to high age, diarrhea, or gut inflammation $(18,42)$. In a large series of 108 VHL patients with pNETs in whom the serum tumor markers insulin, glucagon, pancreatic polypeptide, and vasoactive intestinal peptide were evaluated, none were indicative of pNET in VHL [6]. This indicates that the measurement of these tumor markers is likely of no value.

In general, small pNET lesions in VHL are of no threat (43). Still, VHL patients die due to advanced pNET (43), indicating that timing of surgery in VHL is not optimal. EUS can be combined with FNA to obtain cytology for NET confirmation and is safe. An earlier study in patients with pNETs, complications due to EUS+FNA occurred in 1\% (44). Moreover, in a pooled analysis of data from 19 EUS centers in the United States, the incidence of pancreatitis after EUS+FNA of solid pancreatic lesions masses was $0.3 \%$ (45). In conclusion, EUS performs well in early detection of pNETs, but its role in VHL surveillance is unclear.

\section{Supplementary data}

This is linked to the online version of the paper at http://dx.doi.org/10.1530/ EJE-15-1012.

\section{Declaration of interest}

All authors declare that there is no conflict of interest that could be perceived as prejudicing the impartiality of the research reported.

\section{Funding}

This study was supported by a Grant of the Dutch Cancer Society (RUG 2008-4188).

\section{Acknowledgments}

The authors thank Drs D. J. Gouma and D. O'Toole for their contributions as members of the external monitoring committee. All authors had access to the study data and had reviewed and approved the final manuscript.

\section{References}

1 Yao JC, Eisner MP, Leary C, Dagohoy C, Phan A, Rashid A, Hassan M \& Evans DB. Population-based study of islet cell carcinoma. Annals of Surgical Oncology 200714 3492-3500. (doi:10.1245/s10434-007-9566-6)

2 Zhou J, Enewold L, Stojadinovic A, Clifton GT, Potter JF, Peoples GE \& $\mathrm{Zhu} \mathrm{K}$. Incidence rates of exocrine and endocrine pancreatic cancers in the United States. Cancer Causes and Control 201021 853-861. (doi: 10.1007/s10552-010-9512-y)
3 Lonser RR, Glenn GM, Walther M, Chew EY, Libutti SK, Linehan WM \& Oldfield EH. Von Hippel-Lindau disease. Lancet 2003361 2059-2067. (doi:10.1016/S0140-6736(03)13643-4)

4 Wilding A, Ingham SL, Lalloo F, Clancy T, Huson SM, Moran A \& Evans DG. Life expectancy in hereditary cancer predisposing diseases: an observational study. Journal of Medical Genetics 2012 49 264-269. (doi:10.1136/jmedgenet-2011-100562)

5 Hammel PR, Vilgrain V, Terris B, Penfornis A, Sauvanet A, Correas JM, Chauveau A, Correas JM, Chauveau D, Balian A et al. Pancreatic involvement in von Hippel-Lindau disease. The Groupe Francophone d'Etude de la Maladie de von Hippel-Lindau. Gastroenterology 2000119 1087-1095.

6 Blansfield JA, Choyke L, Morita SY Blansfield JA, Choyke L, Morita SY, Choyke PL, Pingpank JF, Alexander HR, Seidel G et al. Clinical genetic and radiographic analysis of 108 patients with von Hippel-Lindau disease (VHL) manifested by pancreatic neuroendocrine neoplasms (PNETs). Surgery 2007142 814-818

7 Neuzillet C, Vullierme MP, Coulevard A, Sauvanet A, Levy P, Richard S, Ruzniewski P \& Hammel P. Difficult diagnosis of atypical cystic pancreatic lesions in von Hippel-Lindau disease. Journal of Computer Assisted Tomography 201034 140-145.

8 Kitano M, Millo C, Rahbari R, Herscovitch P, Gesuwan K, Webb RC, VenkatesanAM, Phan GQ, Hughes MS, Libutti SK et al. Comparison of 6-18F-fluoro-L-DOPA, 18F-2-deoxy-D-glucose, CT and MRI in patients with pancreatic neuroendocrine neoplasms with von Hippel-Lindau disease. Surgery 2011150 1122-1128. (doi:10.1016/j. surg.2011.09.048)

9 Rösch T, Lightdale CJ, Botet JF, Boyce GA, Sivak MV Jr, Yasuda K, Heyder N, Palazzo L, Dancygier H, Schusdziarra V et al. Localization of pancreatic endocrine tumors by endoscopic ultrasonography. New England Journal of Medicine 1992326 1721-1726.

10 Langer P, Kann PH, Fendrich V, Richter G, Diehl S, Rothmund M \& Bartsch DK. Prospective evaluation of imaging procedures for the detection of pancreaticoduodenal endocrine tumors in patients with Multiple Endocrine Neoplasia Type 1. World Journal of Surgery 200428 1317-1322.

11 Kann P, Balakina E, Ivan D, Bartsch DK, Meyer S, Klose KJ, Behr T $\&$ Langer P. Natural course of small, asymptomatic neuroendocrine pancreatic tumours in Multiple Endocrine Neoplasia Type 1: an endoscopic ultrasound imaging study. Endocrine-Related Cancer 2006 13 1195-1202. (doi:10.1677/erc.1.01220)

12 Hellman P, Hennings J, Akerström G \& Skogseid B. Endoscopic ultrasonography for evaluation of pancreatic tumours in multiple endocrine neoplasia type 1. British Journal of Surgery 200592 1508-1512.

13 Thomas-Marques L, Murat A, Delemer B, Penfornis A, Cardot-Bauters C, Baudin E, Niccoli-Sire P, Levoir D, ChoplinHdu B, Chabre O, Jovenin N, Cadiot G \& Groupe des Tumeurs Endocrines (GTE). Prospective endoscopic ultrasonographic evaluation of the frequency of nonfunctioning pancreaticoduodenal endocrine tumors in patients with multiple endocrine neoplasia type 1. American Journal of Gastroenterology $2006 \mathbf{1 0 1} 266-273$.

14 Lewis MA, Thompson GB \& Young WF Jr. Preoperative assessment of the pancreas in multiple endocrine neoplasia type 1. World Journal of Surgery 201236 1375-1381. (doi:10.1007/s00268-012-1539-7)

15 van Asselt SJ, Brouwers AH, van Dullemen HM, van der Jagt EJ, Bongaerts AH, Kema IP, Koopmans KP, Valk GD, Timmers HJ, de Herder WW et al. EUS is superior for detection of pancreatic lesions compared with standard imaging in patients with multiple endocrine neoplasia type 1. Gastrointestinal Endoscopy 201581 159-167. (doi:10.1016/j.gie.2014.09.037)

16 Sundin A, Eriksson B, Bergström M, Bjurling P, Lindner KJ, Oberg K \& Långström B. Demonstration of [11C]-5-hydroxy-L-tryptophan uptake and decarboxylation in carcinoid tumors by specific positioning labeling in positron emission tomography. Nuclear Medicine and Biology 200027 33-41. 
17 Koopmans KP, Neels OC, Kema IP, Elsinga PH, Sluiter WJ, Vanghillewe K, Brouwers AH, Jager PL \& de Vries EG. Improved staging of patients with carcinoid and islet cell tumors with 18F-dihydroxy-phenyl-alanine and 11C-5-hydroxy-tryptophan positron emission tomography. Journal of Clinical Oncology 200826 1489-1495. (doi:10.1200/JCO.2007.15.1126)

18 Modlin IM, Gustafsson BI, Moss SF, Pavel M, Tsolakis AV \& Kidd M. Chromogranin A - biological function and clinical utility in neuroendocrine tumor disease. Annals of Surgical Oncology 201017 2427-2443. (doi:10.1245/s10434-010-1006-3)

19 Reznek RH. CT/MRI of neuroendocrine tumours. Cancer Imaging 2006 6 S163-S177. (doi:10.1102/1470-7330.2006.9037)

20 Balon HR, Goldsmith SJ, Siegel BA, Silberstein EB, Krenning EP, Lang $\mathrm{O}$, Donohoe KJ \& Society of Nuclear Medicine. Procedure guideline for somatostatin receptor scintigraphy with (111)In-pentetreotide. Journal of Nuclear Medicine 200142 1134-1138.

21 Iglesias-Garcia J, Larino-Noia J, Abdulkader I, Forteza J, DominguezMunoz J. EUS elastography for the characterization of solid pancreatic masses. Gastrointestinal Endoscopy 200970 1101-1108. (doi:10.1016/j. gie.2009.05.011)

22 Bonta PI, Kok MF, Bergman JJ, Van den Brink GR, Lemkes JS, Tytgat GN \& Fockens P. Conscious sedation for EUS of the esophagus and stomach: a double-blind, randomized, controlled trial comparing midazolam with placebo. Gastrointestinal Endoscopy 200357 842-847.

23 Compton CC. Serous cystic tumors of the pancreas. Seminars in Diagnostic Pathology 200017 43-55.

24 Gabata T, Terayama N, Yamashiro M, Takamatsu S, Yoshida K, Matsui O, UsukuraM, Takeshita M \& Minato H. Solid serous cystadenoma of the pancreas: MR imaging with pathologic correlation. Abdominal Imaging 200530 605-609.

25 Takeshita K, Kutomi K, Takada K, Kohtake H, Furui S, Takada T \& Fukushima J. Unusual imaging appearances of pancreatic serous cystadenoma: correlation with surgery and pathologic analysis. Abdominal Imaging 200530 610-615.

26 Gerke H, Silva R \& Jensen CS. Hypervascular pancreatic tumor diagnosed as a serous cystadenoma by EUS-guided Trucut biopsy. Gastrointestinal Endoscopy $200564273-274$.

27 Voss M, Hammel P, Molas G, Palazzo L, Dancour A, O'Toole D, Terris B, Degott C, Bernades P, Ruszniewski P et al. Value of endoscopic ultrasound guided fine needle aspiration biopsy in the diagnosis of solid pancreatic masses. Gut 200046 244-249.

28 Ardengh JC, de Paulo GA, Ferrari AP. EUS-guided FNA in the diagnosis of pancreatic neuroendocrine tumors before surgery. Gastrointestinal Endoscopy $200460378-384$.

29 Figueiredo FA, Giovannini M, Monges G, Charfi S, Bories E, Pesenti C, Caillol F \& Delpero JR. Pancreatic endocrine tumors: a large single-center experience. Pancreas 200938 936-940. (doi:10.1097) MPA.0b013e3181b365db)

30 Affolter KE, Schmidt RL, Matynia AP, Adler DG \& Factor RE. Needle size has only a limited effect on outcomes in EUS-guided fine needle aspiration: a systematic review and meta-analysis. Digestive Diseases and Sciences 201358 1026-1034. (doi:10.1007/s10620-012-2439-2)

31 Larghi A, Capurso G, Garnuccio A et al. Ki-67 grading of nonfunctioning pancreatic neuroendocrine tumors on histologic samples obtained by EUS-guided fine-needle tissue acquisition: a prospective study. Gastrointestinal Endoscopy 201276 570-577. (doi:10.1016/j.gie.2012.04.477)

32 Orlefors H, Sundin A, Ahlström H, Bjurling P, Bergström M, Lilja A, Långström B, Oberg $\mathrm{K} \&$ Eriksson B. Positron emission tomography with 5-hydroxytryprophan in neuroendocrine tumors. Journal of Clinical Oncology 199816 2534-2541.
33 Eisenhofer G. Screening for pheochromocytomas and paragangliomas. Current Hypertension Reports 201214 130-137.

34 Becker I, Paulus W \& Roggendorf W. Histogenesis of stromal cells in cerebellar hemangioblastomas. American Journal of Pathology 1989134 271-275.

35 Ronkainen H, Soini Y, Vaarala MH, Kauppila S \& Hirvikoski P. Evaluation of neuroendocrine markers in renal cell carcinoma. Diagnostic Pathology 20105 28. (doi:10.1186/1746-1596-5-28)

36 Corcos O, Couvelard A, Giraud S, Vullierme MP, Dermot O,Toole, ReboursV, Stievenart JL, Penfornis A, Niccoli-Sire P, Baudin E et al. Endocrine pancreatic tumors in von Hippel-Lindau disease: clinical, histological and genetic features. Pancreas 200837 85-93. (doi:10.1097/MPA.0b013e31815f394a)

37 Bombardieri E, Ambrosini V, Aktolun C, Baum RP, Bishof-Delaloye A, Del Vecchio S, Maffioli L, Mortelmans L, Oyen W, Pepe G et al. 111In-pentetreotide scintigraphy: procedure guidelines for tumour imaging. European Journal of Nuclear Medicine and Molecular Imaging 201037 1441-1448.

38 Gabriel M, Decristoforo C, Kendler D, Dobrozemsky G, Heute D, Uprimny C, Kovacs P, Von Guggenberg E, Bale R \& Virgolini IJ. 68Ga-DOTA-Tyr3-octreotide PET in neuroendocrine tumors: comparison with somatostatin receptor scintigraphy and CT. Journal of Nuclear Medicine 200748 508-518.

39 Buchmann I, Henze M, Engelbrecht S, Eisenhut M, Runz A, Schäfer M, Schilling T, Haufe S, Herrmann T, Haberkorn U et al. Comparison of 68Ga-DOTATOC PET and 111In-DTPAOC (Octreoscan) SPECT in patients with neuroendocrine tumours. European Journal of Nuclear Medicine and Molecular Imaging 200734 1617-1626.

40 Schmid-Tannwald C, Schmid-Tannwald CM, Morelli JN, Neumann R, Haug AR, Jansen N, Nikolaou K, Schramm N, Reiser MF, Rist $\mathrm{C}$ et al. Comparison of abdominal MRI with diffusion-weighted imaging to 68Ga-DOTATATE PET/CT in detection of neuroendocrine tumors of the pancreas. European Journal of Nuclear Medicine and Molecular Imaging 201340 897-907. (doi:10.1007/s00259-013-2371-5)

41 Farchione A, Rufini V, Brizi MG, Iacovazzo D, Larghi A, Massara RM, Petrone G, Poscia A, Treglia G, De Marinis L et al. Evaluation of the added value of diffusion-weighted imaging to conventional magnetic resonance imaging in pancreatic neuroendocrine tumors and comparison with 68Ga-DOTANOC positron emission tomography/ computed tomography. Pancreas 201545 345-354. (doi:10.1097) MPA.0000000000000461)

42 Oberg K. Circulating biomarkers in gastroenteropancreatic neuroendocrine tumours. Endocrine-Related Cancer 201118 S17-S25. (doi:10.1530/ERC-10-0280)

43 de Mestier L, Gaujoux S, Cros J, Hentic O, Vullierme MP, Couvelard A, Cadiot G, Sauvanet A, Ruszniewski P, Richard S et al. Long-term prognosis of resected pancreatic neuroendocrine tumors in von Hippel-Lindau disease is favorable and not influenced by small tumors left in place. Annals of Surgery $20152 \mathbf{2 6 2}$ 384-388. (doi:10.1097/SLA.0000000000000856)

44 Atiq M, Bhutani MS, Bektas M, Lee JE, Gong Y, Tamm EP, Shah $\mathrm{CP}$, Ross WA, Yao J, Raju GS et al. EUS-FNA for pancreatic neuroendocrine tumors: a tertiary cancer center experience. Digestive Diseases and Sciences 201257 791-800. (doi:10.1007/ s10620-011-1912-7)

45 Eloubeidi MA, Gress FG, Savides TJ, Wiersema MJ, Kochman ML, Ahmad NA, Ginsberg GG, Erickson RA, Dewitt J, Van Dam J et al. Acute pancreatitis after EUS-guided FNA of solid pancreatic masses: a pooled analysis from EUS centers in the United States. Gastrointestinal Endoscopy $200460385-389$.

Received 12 October 2015

Revised version received 10 January 2016

Accepted 15 February 2016 